\title{
Process analytical technology (PAT) approach to the formulation of thermosensitive protein-loaded pellets: Multi-point monitoring of temperature in a high-shear pelletization
}

\author{
Katalin Kristó a , Orsolya Kovács ${ }^{\mathrm{a}}$, András Kelemen ${ }^{\mathrm{b}}$, Ferenc Lajkó ${ }^{\mathrm{c}}$, Gábor Klivényi ${ }^{\mathrm{c}}$, Béla Jancsik ${ }^{\mathrm{c}}$, \\ Klára Pintye-Hódi ${ }^{a}$, Géza Regdon jr. a,* \\ a Department of Pharmaceutical Technology, University of Szeged, Eötvös u. 6, 6720 Szeged, Hungary \\ b Department of Applied Informatics, University of Szeged, Boldogasszony sgt. 6, 6725 Szeged, Hungary \\ c Opulus Ltd., Fürj u. 92/B, 6726, Szeged, Hungary
}

\section{A R T I C L E I N F O}

Article history:

Received 15 February 2016

Received in revised form 25 August 2016

Accepted 26 August 2016

Available online $\mathrm{xxxx}$

\section{Keywords:}

High-shear pelletization

Elevated temperature measurement

Protein

Pepsin

Enzyme activity

Process analytical technology (PAT)

\begin{abstract}
A B S T R A C T
In the literature there are some publications about the effect of impeller and chopper speeds on product parameters. However, there is no information about the effect of temperature. Therefore our main aim was the investigation of elevated temperature and temperature distribution during pelletization in a high shear granulator according to process analytical technology. During our experimental work, pellets containing pepsin were formulated with a high-shear granulator. A specially designed chamber (Opulus Ltd.) was used for pelletization. This chamber contained four PyroButton-TH® sensors built in the wall and three PyroDiff $\circledast$ sensors 1,2 and $3 \mathrm{~cm}$ from the wall. The sensors were located in three different heights. The impeller and chopper speeds were set on the basis of $3^{2}$ factorial design. The temperature was measured continuously in 7 different points during pelletization and the results were compared with the temperature values measured by the thermal sensor of the high-shear granulator. The optimization parameters were enzyme activity, average size, breaking hardness, surface free energy and aspect ratio. One of the novelties was the application of the specially designed chamber (Opulus Ltd.) for monitoring the temperature continuously in 7 different points during high-shear granulation. The other novelty of this study was the evaluation of the effect of temperature on the properties of pellets containing protein during high-shear pelletization.
\end{abstract}

(c) 2016 Elsevier B.V. All rights reserved.

\section{Introduction}

Biologically active peptides and proteins are increasingly becoming a very important class of therapeutic agents because of their extremely specific activity and high tolerability by the human organism. Pepsin, the main digestive enzyme in gastric juice, is responsible for the most of digestive activities in the stomach (Zeng et al., 2014). Typical therapeutic uses of pepsin involve pathological states accompanied by hypo- or anacidity, such as Sjögren's syndrome.

The stability of enzymes is one of the most difficult problems in pharmaceutical technology, in consequence of the great number of factors involved (Simon et al., 2007). However, during processing into solid dosage forms, e.g. by the use of pelletization, enzyme activity can decrease because of the mechanical effects, moisture content and increased temperature that can arise in the course of high-shear pelletization (Kristó and Pintye-Hódi, 2013).

\footnotetext{
* Corresponding author.

E-mail address: geza.regdon@pharm.u-szeged.hu (G. Regdon).
}

Most high-shear mixers consist of a stainless steel vessel, an impeller and a chopper. Many authors (Benali et al., 2009; Chitu et al., 2011; Rahmanian et al., 2011) have studied the effect of impeller speed on granule/pellet properties. Diverse influences have been found: from almost independent granule/pellet size distributions (Rahmanian et al., 2011) to significantly affected granule/pellet size distributions in a way of reducing entity sizes (Chitu et al., 2011; Zizek et al., 2013).

Quality control by the pharmaceutical industry has traditionally relied on the assessment of the raw materials prior to processing and the analytical determinations of the end-product (Kaneko et al., 2015). This tradition has the inherent characteristics of poor efficiency, unnecessary quality risk and high cost. The Food and Drug Administration (FDA) initiative urges the industry to employ innovation, cutting edge scientific and engineering knowledge, along with the best principles of quality management to respond to the challenges to provide cost effective medicine at the highest quality and the lowest possible risk to the patient.

Reliable technology processes demand an understanding of the pelletization processes, as well as identification and application of the critical factors that determine the pelletization quality. The ICH 
(International Conference on Harmonisation of Technical Requirements for Registration of Pharmaceuticals for Human Use) Q8 (Pharmaceutical Development); guidelines emphasize the adoption of quality by design (QbD) (ICH Q8, 2009; Ferreire and Tobyn, 2015; Fonteyne et al., 2014; Djokica et al., 2014, Shah et al., 2015) in the development of pharmaceutical products. This definition can found in ICH Q8 guideline: "A systematic approach to development that begins with predefined objectives and emphasizes product and process understanding and process control, based on sound science and quality risk management."(ICH Q8, 2009). QbD means designing and developing a product and associated manufacturing processes that will be used during product development to ensure that the product consistently attains a predefined quality at the end of the manufacturing process.

In 2002, the FDA encouraged the use of process analytical technology (PAT) by the pharmaceutical industry. PAT is intended to assure product quality via meaningful design, monitoring, control and surveillance of each manufacturing stage. With this methodology, quality in the product and efficiency in the production process result from a deep knowledge of the process and strict control of any physical, chemical and quality-related factors influencing each stage. Quality in pharmaceutical production processes cannot be assured merely by analysing raw materials and end-products; rather, it requires meaningful design and implementation in each production stage (Guidance for Industry, 2004; Bakeev, 2010; Carnedas et al., 2015). The definition of PAT is in the European Pharmacopoeia (Ph. Eur.). This definition is the following:

"Process analytical technology (PAT): a system for designing, analysing and controlling manufacturing through timely measurements (i.e. during processing) of critical quality and performance attributes of raw and in-process materials and processes with the goal of ensuring final product quality" (European Pharmacopoeia 8.4, 5.15).

PAT (Lopesa et al., 2004; Roggo et al., 2007, Bu et al., 2013; Hansuld and Briens, 2014; Hudovornik et al., 2015) plays an important role in the pharmaceutical industry. PAT is used extensively in process development, process understanding and process control (Reid et al., 2012). A process is generally considered well understood when (1) all critical sources of variability are identified and explained; (2) variability is managed by the process; and (3) product quality attributes can be accurately and reliably predicted over the design space established for materials used, process parameters, manufacturing, environmental, and other conditions.

Although a few authors have demonstrated the use of multivariate near infrared (NIR) chemometric models coupled with temperature and humidity data to develop models for better understanding the granulation and coating process (Rantanen et al., 2000; Rantanen et al., 2001), the use of PyroButton-TH® (made by Opulus Ltd.) calibrated sensors (ISO 17025) for high-shear pelletization was never explored. These data loggers were reported for use in sterilization validation and monitoring in steam sterilization, humidity and temperature monitoring of environment and facilities such as warehouse, and processing procedures in pharmaceutical technology (Pandey et al., 2014a, 2014b; Kestur et al., 2014; Macchi et al., 2016). However, there is no reported use of this high-shear pelletization. These data loggers can be programmed for time and the duration of data collection. Due to their small size ( $17 \mathrm{~mm} \times 6 \mathrm{~mm}$ size), they can be placed at various locations in and around the granulator for accurate monitoring. These loggers are self-powered by a lithium battery and internally consist of a microprocessor, a capacitive humidity sensor and a quartz clock, all enclosed in a hermetically sealed stainless steel housing, hence these data loggers can be chemically sterilized and depyrogenated and each data logger's calibration is National Institute of Standards and Technology (NIST) traceable (Kona et al., 2013).

Our main aim was to investigate the effects of the elevated temperature on the parameters of the product. During pelletization, the sample can be exposed to considerable mechanical effects and elevated temperatures, and during high-shear pelletization the impeller and chopper
Table 1

Standard parameters of the granulation process.

\begin{tabular}{ll}
\hline Amount of granulation liquid $(\mathrm{ml})$ & 96 \\
\hline Amount of pepsin $(\mathrm{g})$ & 4 \\
Amount of Vivapur 101 $(\mathrm{g})$ & 96 \\
Dosing speed of granulation liquid $(\mathrm{ml} / \mathrm{min})$ & 5 \\
Process time $(\mathrm{s})$ & 1152 \\
Rounding time $(\mathrm{s})$ & 60 \\
\hline
\end{tabular}

speeds can induce elevated temperatures and influence the parameters of the products (Luukkonen et al., 2008).

This information can deepen the understanding of the effects of different technological processes, which is indispensable for the determination of the critical control points in the preparation of solids containing proteins. The preservation of the enzyme activity level of pepsin and other proteins should be taken into consideration during the formulation.

\section{Materials and methods}

\subsection{Materials}

In the course of the experimental work, purified water (distilled water, Ph. Eur.) and microcrystalline cellulose (MCC; Vivapur 101, J. Rettenmaier \& Söhne GmbH + Co., Rosenberg, Germany) (D50 65 m; bulk density $0.29 \mathrm{~g} / \mathrm{cm} 3$; data from producer) were used. The active pharmaceutical ingredient was porcine pepsin powder ( $\mathrm{Ph}$. Eur., Meditop Ltd., Pilisborosjenő, Hungary) (D50 $10 \mu \mathrm{m}$ ). Bovine haemoglobin (SigmaAldrich), Folin-Ciocalteu reagent (Sigma-Aldrich), trichloroacetic acid (Molar Chemicals Ltd.), hydrochloric acid (Ph. Eur.) and sodium hydroxide (Ph. Eur.) were used for pepsin activity measurement.

\subsection{Methods}

\subsubsection{Preparation of samples}

The granulating fluid was distilled water $(96 \mathrm{ml})$. The powder mixture contained pepsin powder ( $4 \mathrm{~g}$ ) and Vivapur 101 (96 g). The powder was homogenized with a Turbula mixer (Willy A. Bachofen Maschinenfabrik, Basel, Switzerland) for $10 \mathrm{~min}$.

The wet granulating process was carried out in a ProCepT 4M8 highshear granulator (ProCepT nv, Zelzate, Belgium). The impeller and chopper were both positioned vertically in the high-shear granulator, and their speeds were taken as the factors in the factorial design, chosen on the basis of the preformulation studies. The other parameters were standard (Table 1).

The temperature was continuously monitored by the specially designed chamber with PyroButton-TH®) and PyroDiff ${ }^{\circledR}$ (Opulus Ltd.; Hungary) sensors and the high-shear granulator's thermal sensor. The granules were dried at room temperature $\left(25 \pm 1{ }^{\circ} \mathrm{C}\right)$ for $24 \mathrm{~h}$.

\subsubsection{Factorial design}

The factorial design is a suitable method for modelling and predicting the effects of the technological parameters (the chopper and impeller speeds) on the pepsin properties. The $3^{2}$ factorial design was applied (Table 2).

The high levels were based on the technical parameters of the highshear granulator, and the low levels on the results of the preformulation studies. The variation interval was the range between the high and low

Table 2

The optimization parameters were average particle size and enzyme activity.

\begin{tabular}{llll}
\hline & Low level & Zero level & High level \\
\hline Impeller (rpm) & 300 & 900 & 1500 \\
Chopper (rpm) & 500 & 2750 & 5000 \\
\hline
\end{tabular}


levels. The zero level was calculated as the arithmetic mean of the high and low levels.

The experiment was based on a $3^{2}$ full factorial design, with the equation

$$
\begin{aligned}
y= & b_{0}+b_{1}(L) \times x_{1}+b_{1}(Q) x_{1}{ }^{2}+b_{2}(L) x_{2}+b_{2}(Q) x_{2}{ }^{2}+b_{1}(L)_{2}(Q) x_{1} x_{2} \\
& \left.+b_{1}(L)_{2}(Q) x_{1} x_{2}{ }^{2}+b_{1}(Q)_{2}(L) x_{1}{ }^{2} x_{2}+b_{1}(Q)\right)_{2}(Q) x_{1}{ }^{2} x_{2}{ }^{2}
\end{aligned}
$$

where

$y$ : the optimization parameter.

$b_{0}$ : the average optimization parameter value.

$b_{1}$ : a coefficient describing the effect of the chopper speed.

$b_{2}$ : a coefficient describing the effect of the impeller speed.

$b_{12}$ : a coefficient describing the effect of the interaction of the chopper and impeller speeds.

Q: quadratic part.

L: linear part.

$x_{1}$ : the chopper speed.

$x_{2}$ : the impeller speed.

Statistica for Windows 12 AGA software (StatSoft Inc., Tulsa, USA) was used for the statistical evaluation of the results. This software can calculate the coefficients $\left(b_{0}, b_{1}, b_{2}, b_{12}\right)$, too.

The following model parameters were chosen in Statistica for Windows 12:

$3^{* *}(2-0)$ full factorial design

factors/blocks/runs: 2/1/9

- Include in model: 2-way interactions (linear, quadratic)

aNOVA error term: SS residual

- Confidence interval: $95 \%$
2.2.3. Measurement temperature with PyroDiff® and PyroButton-TH® sensors

The novel, commercially available data loggers known as PyroButton-TH® and PyroDiff ${ }^{\circledR}$ sensors (Opulus Ltd., Hungary) were used in this study for temperature and humidity measurement at different regions of the specially designed chamber by Opulus Ltd. for a highshear granulator (Fig. 1). Specification of PyroButton-TH ${ }^{\circledR}$ and PyroDiff®:

Temperature range: $-20-85^{\circ} \mathrm{C}$

Temperature resolution: $0.0625^{\circ} \mathrm{C}$

Humidity resolution: $0.04 \% \mathrm{RH}$

Data acquisition time: $1 \mathrm{~s}-273 \mathrm{~h}$

Max. Uncertainty: $\pm 0.38^{\circ} \mathrm{C}$

Max. Uncertainty: $\pm 2 \% \mathrm{RH}$

The infrared thermometer of ProCepT 4 M8 granulator can measure in a temperature range of $20-100{ }^{\circ} \mathrm{C}$. This sensor was not calibrated, and other information was not given by the producer. The powder contamination and the vapour can influence the temperature measurement

The effects of the impeller and chopper speeds on pepsin activity and the average particle size were investigated according to a $3^{2}$ factorial design.

\subsubsection{Average particle size (D50) and size distribution}

The average particle size and the size distribution of the granules were measured by a laser diffractometer in dry mode (Mastersizer S, Malvern Instruments Ltd., Worcestershire, UK). Three parallel measurements were performed. Laser diffractometry yields the volume size distribution, with particle measurement in the size range $0.1-2000 \mu \mathrm{m}$ and around $0.5 \mathrm{~g}$ of granules is necessary for the measurement.

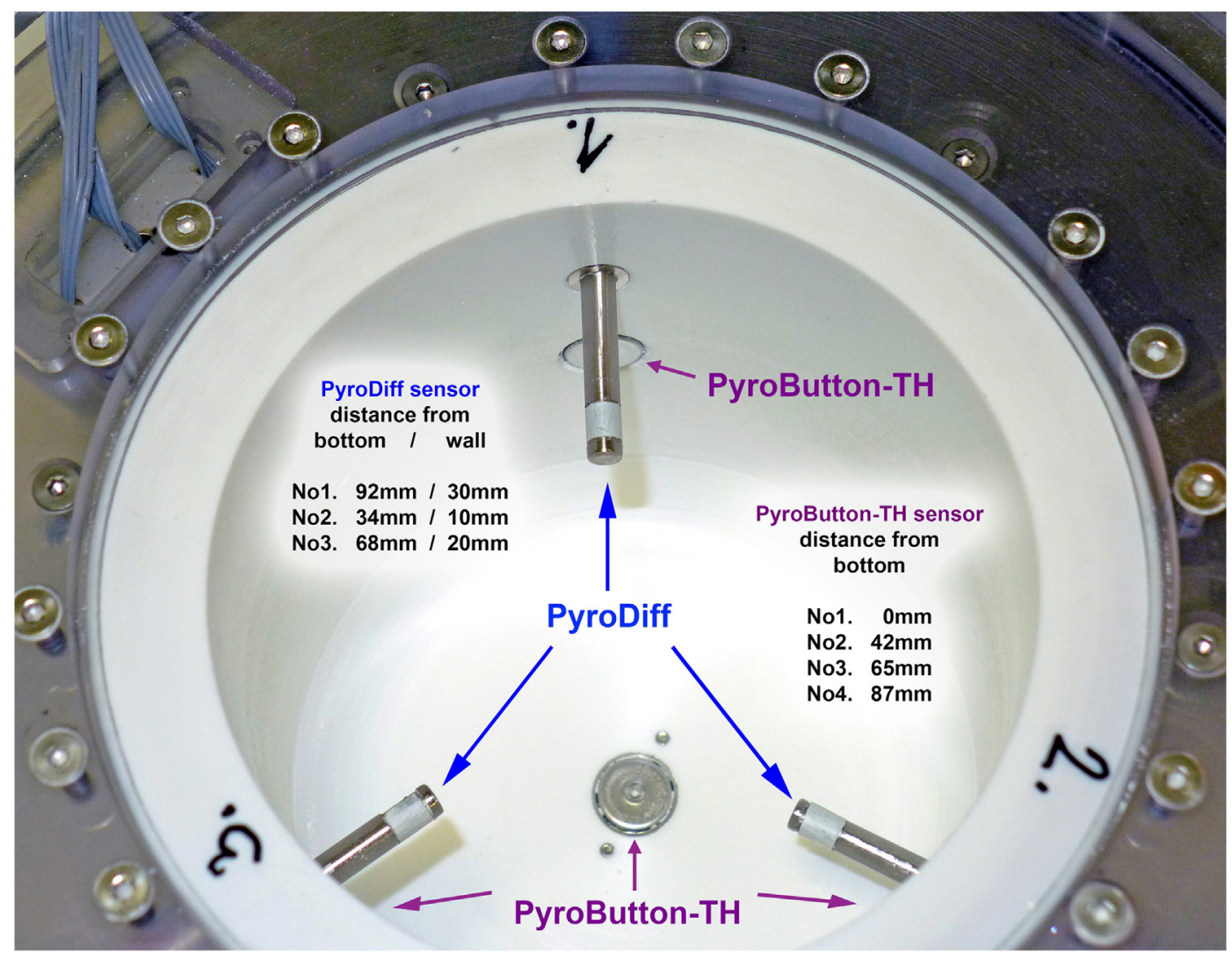

Fig. 1. The specially designed chamber by Opulus Ltd. 


\subsubsection{Determination of enzyme activity}

The $A$ values of the samples relative to the substrate bovine haemoglobin were measured according to the Ph. Eur. The basis of the analysis was the measurement of the amount of protein which could not be precipitated with trichloroacetic acid. Haemoglobin was dissolved at $2 \%$ in $0.1 \mathrm{M}$ hydrochloric acid solution, and the $\mathrm{pH}$ was adjusted to $1.6 \pm 0.1$. The samples and the untreated pepsin powder were dissolved at $0.25 \%$ in $0.1 \mathrm{M}$ hydrochloric acid, and the $\mathrm{pH}$ was adjusted to $1.6 \pm 0.1$. Incubation was performed for $10 \mathrm{~min}$ at $25^{\circ} \mathrm{C}$. $4 \%$ trichloroacetic acid solution was used to precipitate the proteins. The samples were filtered twice through filter paper, leached with $5 \mathrm{ml}$ of trichloroacetic acid and dried. After dilution, $1 \mathrm{ml}$ of ( $5 \mathrm{M}$ ) sodium hydroxide and $1 \mathrm{ml}$ of Folin-Ciocalteu reagent were added as colour-producing reagent, and the solution was left to stand for $15 \mathrm{~min}$ at room temperature. The relative A was determined; the A value of the untreated pepsin was taken as $100 \%$. The amount of non-precipitating protein was determined at $540 \mathrm{~nm}$ with a UV spectrophotometer (Unicam Helios Alpha, Spectronic Unicam, Cambridge, UK).

\subsubsection{Mechanical property}

The breaking hardness was tested for granules which were in the $630-1120 \mu \mathrm{m}$ range of pellet size. This device contains a special specimen holder and a stamp, and it is connected to a computer via an interface; thus, not only can the ultimate deformation force be measured, but the process (force-time and force-displacement curves) can also be followed. If the measured plot (force-time) is parallel to the x-axis, the deformation is viscoelastic; if the plot rises linearly, the deformation is elastic. The specimen is located horizontally and the stamp moves vertically (Fig. 2). Twenty parallel measurements were performed. The measuring range was $0-200 \mathrm{~N}$, the speed of the stamp was $20 \mathrm{~mm} /$ min, the output was $0-5 \mathrm{~V}$, and the sensitivity was $\pm 0.5 \% \pm 0.1$ digit. The sensor was UNICELL force measuring equipment, calibrated with the C9B $20 \mathrm{kN}$ cell.

\subsubsection{Calculation of surface free energy}

The microstructure of the pellet surface was predicted from the spreading coefficient, which was calculated from the surface free energy. An indirect method of assessing the surface free energy $(\gamma)$ from wettability measurements is widely used (Buckton, 1997; Bajdik et al., 2007). In the method of $\mathrm{Wu},(\mathrm{Wu}, 1971)$ the surface free energy is

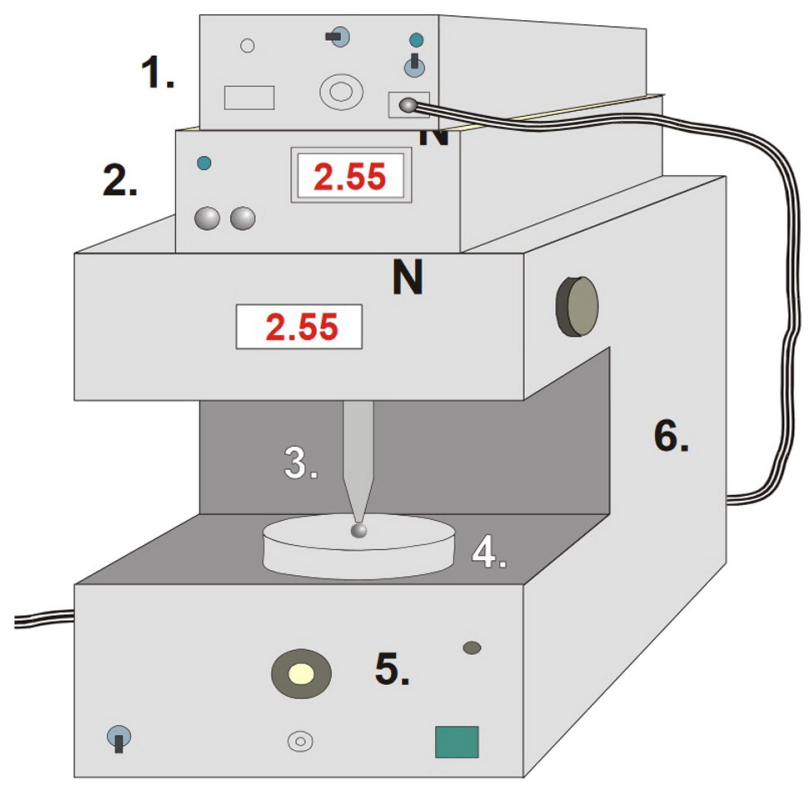

Fig. 2. Breaking hardness tester (1: DAQ unit; 2: current force display; 3: pressure jowl; 4: sample holder; 5 : force measurement unit; 6 : motor and analogue velocity control for pressure jowl). taken as the sum of dispersive $(\gamma d)$ and polar ( $\gamma p)$ components. The surface free energy of solid materials can be determined by means of contact angle measurements with two different liquids with known polar and disperse part of surface tension properties. They can be assessed by solving an equation with two unknowns:

$(1+\cos \Theta) \gamma 1=\frac{4\left(\gamma_{s}^{d} \gamma_{l}^{d}\right)}{\gamma_{s}^{d}+\gamma_{l}^{d}}+\frac{4\left(\gamma_{s}^{p} \gamma_{l}^{p}\right)}{\gamma_{s}^{p}+\gamma_{l}^{p}}$

where $\theta$ is the contact angle, $\gamma_{s}$ is the solid surface free energy and $\gamma_{1}$ is the liquid surface tension [superscripts refer to their polar $\left(\gamma^{\mathrm{P}}\right)$ and dispersive part $\left.\left(\gamma^{\mathrm{d}}\right)\right]$.

If the surface free energy of the solid materials is known, the spreading coefficient $(\mathrm{S})$ may be computed and the interactions between the two materials may be predicted. The spreading coefficient is calculated as the difference between the adhesion work and the cohesion work. The two materials which interact can be two powders, a powder and a liquid, or any material and the equipment. The spreading coefficient $\left(\mathrm{S}_{12}\right)$ of a material "1" over the surface of another material "2" can be determined as follows (Rowe, 1989).

$S_{12}=4\left[\frac{\gamma_{1}^{d} \gamma_{2}^{d}}{\gamma_{1}^{d}+\gamma_{2}^{d}}+\frac{\gamma_{1}^{p} \gamma_{2}^{p}}{\gamma_{1}^{p}+\gamma_{2}^{p}}-\frac{\gamma_{1}}{2}\right]$

An optical contact angle - measuring device (OCA 20, DataPhysics Instruments $\mathrm{GmbH}$, Filderstadt, Germany) was utilized to determine the wetting properties of the samples. The test fluids were distilled water and diiodomethane (Merck KGaA, Darmstadt, Germany). According to Ström, [30] the dispersion part of the surface tension was $21.8 \mathrm{mN} / \mathrm{m}$ for water and $50.8 \mathrm{mN} / \mathrm{m}$ for diiodomethane. The polar part of the surface tension was $51 \mathrm{mN} / \mathrm{m}$ for water and $0 \mathrm{mN} / \mathrm{m}$ for diiodomethane. Compacts of $0.50 \mathrm{~g}$ of pellet were made with a hydraulic press (Specac Inc., Graseby, UK), with a dwell time of $10 \mathrm{~s}$, at a pressure of 10 tons. Circle fitting was applied to determine the contact angle formed on comprimates prepared from different samples.

\subsubsection{Aspect ratio}

The particle size and the shape of the pellets were studied by using a stereomicroscope (Zeiss Stemi 2000-C, Carl Zeiss GmbH, Vienna, Austria). An Image J. image processing and analysis system (Wayne Rasband National Institutes of Health, USA) was used. The aspect ratio was utilized for the evaluation of the shape of the particles. 500 pellets of each sample were checked. The aspect ratio (AR) was calculated with the following equation: $A R=d_{\max } / d_{\min }$, where the longest and shortest diameters were measured.

\section{Results and discussion}

\subsection{Temperature results}

The temperature results were registered during every granulation in 7 different points inside the chamber during pelletization (Fig. 3). This measurement method meets the requirements of PAT. The temperature was measured continuously by the infrared thermometer of ProCepT granulator. These data were compared with the results measured by PyroButton-TH® and PyroDiff $®$ sensors.

The temperature measured by ProCepT infra was lower in every case than the temperature measured by PyroButton-TH® and PyroDiff® sensors. It can be concluded that the ProCepT infra can measure on the surface of the wet masses but the PyroButton-TH $₫$ and PyroDiff $₫$ sensors can measure temperature directly inside the different regions of the chamber. The highest temperature can be measured with PyroButton$\mathrm{TH} \circledast$ on the bottom and the PyroDiff $®$ sensor $10 \mathrm{~mm}$ from the bottom. In the lower region of the chamber the temperature was higher because of the impeller position. The distance is very small between the impeller 


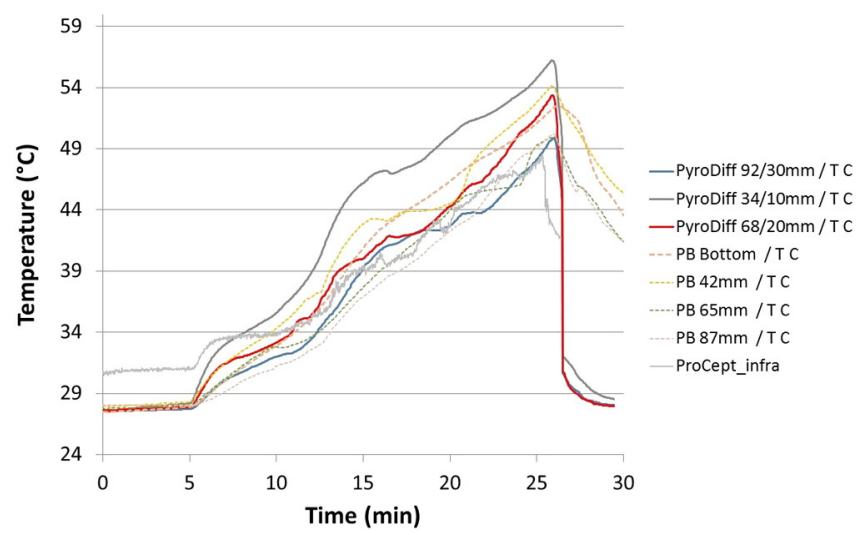

Fig. 3. The temperature results of the maximum impeller - maximum chopper/PB: PyroButton-TH ${ }^{\circledR}$ sensors built in the wall in three different distances from the bottom, one built in the bottom, and the PyroDiff® sensors in three different distances from the bottom, and in three different distances from the wall (the first number is the distance from the bottom, the second number is the distance from the wall).

and the bottom of the chamber, therefore friction is high during granulation. Because of the high friction, the temperature is elevated. In the higher region the temperature was lower. These parameters are very important because on the basis of our previous results (Kristó and Pintye-Hódi, 2013), pepsin activity decreases with temperature increasing in the wet masses. The maximum temperature value measured during every high-shear pelletization was selected. The maximum temperature values are shown in Table 3. The optimization parameters were investigated with maximum temperature.

The chopper speed cannot affect the temperature, only the impeller speed can increase (Fig. 4). There was a logarithmic polynomial with a correlation coefficient value of 0.8542 between the impeller speed and the maximum temperature. Thus the impeller speed is very important not only in respect to pellet parameters, but also regarding elevated temperature. Therefore this parameter and the elevated temperature must be taken into consideration during the formulation of pellets or granules. In the case of thermo-sensitive materials or proteins, the continuous temperature monitoring according to PAT different regions inside the chamber is recommended.

\subsection{Average particle size and size distribution}

On the basis of Malvern Mastersizer analysis, every sample showed a monodisperse size distribution. For example, in Fig. 5 the sample prepared at $300 \mathrm{rpm}$ impeller and $5000 \mathrm{rpm}$ chopper speed can be seen, where the d50 value was $861.29 \mu \mathrm{m}$. Only one peak was detected in the case of every sample. The Span value was calculated from d10, $\mathrm{d} 50$ and d90 values. These measured data can be seen in Table 4. The Span values were between 0.671 and 1.134 . There was no significant difference among the samples (Table 4).

Table 3

The maximum temperature value.

\begin{tabular}{lll}
\hline Factors & \multicolumn{2}{l}{ Maximum temperature $\left({ }^{\circ} \mathrm{C}\right)$} \\
\cline { 1 - 2 } Impeller $(\mathrm{rpm})$ & Chopper $(\mathrm{rpm})$ & \\
\hline 300 & 500 & 27.49 \\
1500 & 500 & 50.28 \\
900 & 500 & 42.54 \\
300 & 5000 & 27.21 \\
1500 & 5000 & 55.47 \\
900 & 5000 & 42.41 \\
300 & 2750 & 31.95 \\
1500 & 2750 & 58.24 \\
900 & 2750 & 56.56 \\
\hline
\end{tabular}

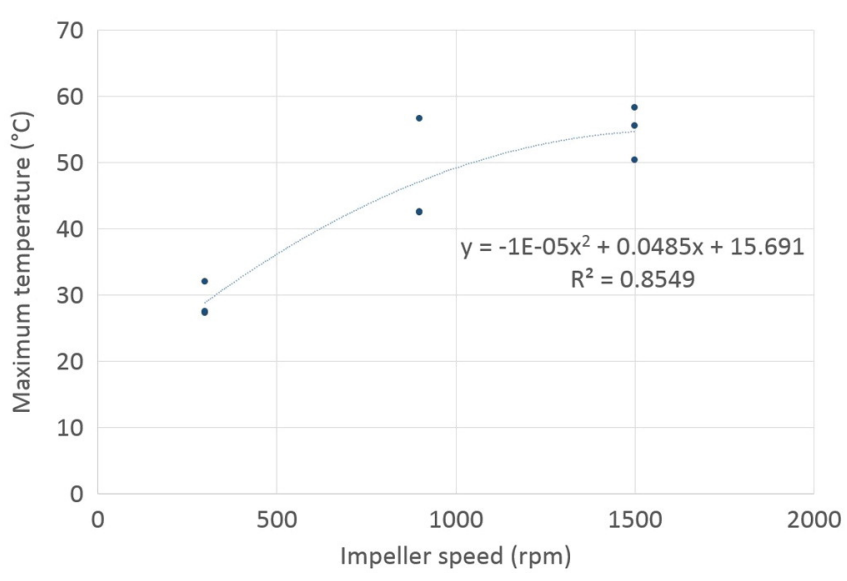

Fig. 4. The maximum temperature value at different impeller speeds.

The effect of impeller and chopper speed on the size of the granules was investigated with the application of factorial design. Both factors and the interaction of the factors were significant $(P<0.05)$, only the " $b_{1}(Q)_{2}(L)$ " interaction was not a significant part (Table 5). The effect of impeller speed was higher than that of chopper speed (Fig. 6). The equation of $\mathrm{d} 50$ of the response surface can be read in Table 6 .

There are some publications in the literature about the effect of impeller speed on the granules/pellet size. Our results correlate with these. Mangwandi et al. (2010) found for water as a binder that the mean size of granules decreases with increasing impeller speed. Benali et al. (2009) determined in the case of between 40 and $150 \mathrm{rpm}$ impeller speed the fine particle that the mean granules diameter increases with increasing impeller speed and in the case of impeller speed beyond $200 \mathrm{rpm}$ the mean granules size decreases with increasing impeller speed. Chitu et al. (2011) also found the granules size will decrease and the percentage of lumps will also decrease for high impeller seed.

The effect of temperature on pellet size was investigated. There was no significant difference in the d50 with increasing temperature (Fig. 7).

\subsection{Enzyme activity}

In the case of protein or enzyme active agents, the enzyme activity measurement is very important because the activity must be preserved in the product. During high-shear granulation, the enzyme activity can decrease because of mechanical stress and elevated temperature.

The equation of the enzyme activity of the response surface can be seen in Table 6 . The enzyme activity measured was between $22 \%$ and $82 \%$. The highest value was in the case of low level-low level factor

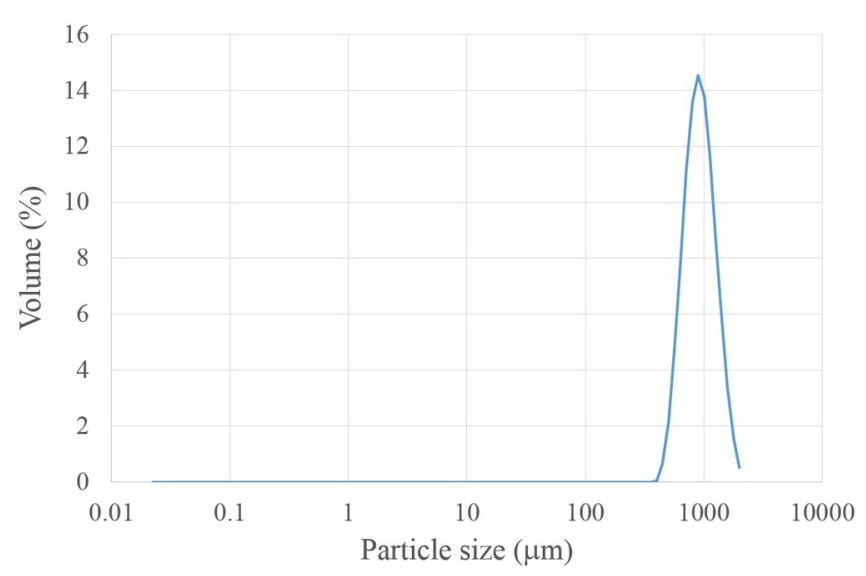

Fig. 5. The result of Malvern Mastersizer analysis of Sample prepared at $300 \mathrm{rpm}$ impeller and $5000 \mathrm{rpm}$ chopper speed. 
Table 4

The results of particle size analysis.

\begin{tabular}{|c|c|c|c|c|}
\hline Impeller (rpm) & Chopper (rpm) & Span & $\mathrm{d} 10(\mu \mathrm{m})$ & $\mathrm{d} 90(\mu \mathrm{m})$ \\
\hline 300 & 500 & $0.832 \pm 0.052$ & $655.14 \pm 35$ & $1496.74 \pm 13.89$ \\
\hline 1500 & 500 & $0.671 \pm 0.004$ & $546.68 \pm 5.57$ & $1054.19 \pm 7.11$ \\
\hline 900 & 500 & $1.134 \pm 0.045$ & $472.08 \pm 23.05$ & $1407.68 \pm 37.24$ \\
\hline 300 & 5000 & $0.842 \pm 0.040$ & $583.41 \pm 4.36$ & $1312.74 \pm 24.97$ \\
\hline 1500 & 5000 & $0.813 \pm 0.110$ & $589.18 \pm 34.75$ & $1290.32 \pm 15.55$ \\
\hline 900 & 5000 & $1.074 \pm 0.006$ & $507.99 \pm 11.65$ & $1427.76 \pm 38.92$ \\
\hline 300 & 2750 & $1.029 \pm 0.002$ & $546.71 \pm 4.62$ & $1485.89 \pm 16.79$ \\
\hline 1500 & 2750 & $0.955 \pm 0.056$ & $571.77 \pm 13.52$ & $1437.99 \pm 22.08$ \\
\hline 900 & 2750 & $0.8 \pm 0.242$ & $705.81 \pm 116.13$ & $1541.67 \pm 5.76$ \\
\hline
\end{tabular}

Span $=($ d90-d10)/d50; d10: 10 th percentile; d90: 90th percentile.

combination (impeller speed: $300 \mathrm{rpm}$; chopper speed: $500 \mathrm{rpm}$ ). In this case, the mechanical stress and the temperature $\left(27.47^{\circ} \mathrm{C}\right)$ were also lower than in other cases. The lowest enzyme activity value was in the case of high level-high level factor combination (impeller speed: $1500 \mathrm{rpm}$; chopper speed: $5000 \mathrm{rpm}$ ) because the mechanical stress and the elevated temperature were higher $\left(55.47^{\circ} \mathrm{C}\right)$.

Both factors and all the interactions were significant. It can be seen in Fig. 6 that because of the higher mechanical stress and higher elevated temperature, the enzyme activity decreased to a greater extent with increasing impeller speed than with increasing chopper speed. In the case of high impeller speed friction is also high, therefore it causes higher mechanical stress and elevated temperature.

It can be seen (Fig. 7) that the enzyme activity decreased linearly with increasing temperature (impeller speed increasing) because pep$\sin$ is also a protein, therefore it is a thermal sensitive material. Thus during the formulation of pellets containing protein the impeller speed must be taken into consideration because of elevated temperature. The other resolution can be the application of cooling with double layer chamber.

\subsection{Surface free energy}

The surface free energy measurement is very important from the aspect of coating because the coating liquid must spread smoothly on the pellet surface. Comprimates from base materials were also prepared and the total surface free energy, the disperse and polar parts of total surface free energy were calculated from the contact angle measurement results (Table 7).

From these data the spreading coefficient was calculated. The spreading coefficient (Material 1: Vivapur 101; Material 2: pepsin) was 5.2. When the sign of the spreading coefficient $\left(S_{12}\right)$ is positive, Material 1 spreads on the surface of Material 2. It can be seen that pepsin covered the Vivapur 101 particles.

The equation of the response surface of total surface free energy can be written with the equation in Table 6 .

There was no significant factor ( only $b_{0}$ ), but it can be seen on the response surface (Fig. 6) if the impeller and chopper speed was high, the total surface free energy was also higher.
It can be observed that the total surface free energy also increased with increasing temperature (Fig. 7). On the basis of the spreading coefficient $\left(S_{12}\right)$, pepsin spread on the Vivapur 101 surface. At higher temperatures this phenomenon is better experienced as at elevated temperatures the total surface free energy of pellets was closer to the surface free energy of pepsin. It can be concluded that at the elevated temperature, the pepsin was more enriched on the surface. The elevated temperature may bring about the unfolding of the protein structure. Our results also support this because the enzyme activity was significantly lower. The increase of total surface free energy with temperature can be explained by the fact that pepsin with an unfolded structure and active pepsin have different properties. The pepsin with an unfolded structure can be enriched on the surface. This information is a very important parameter in terms of coating.

The total surface free energy contains a polar and a disperse part. In this case both factors had a significant effect on the disperse part of surface free energy. In the case of high impeller speed, the disperse part of surface free energy was also high. The equation of the response surface of the disperse part of surface free energy can be read in Table 6.

The polar surface free energy was constant and the disperse part was increased with increasing temperature. From the aspect of coating, the increasing of the disperse part is not favourable because the polar coating liquid cannot spread smoothly on the surface. This phenomenon is undesirable during coating. Therefore the application of high impeller speed cannot be recommended because of coating.

\subsection{The morphology of pellets}

The morphology of pellets was investigated with Image J. software on the basis of the microscopic picture of pellets (Fig. 8). All the samples were similar spherical pellets, as shown in Fig. 8. The software calculated the aspect ratio (AR) from two different diameters. If the AR value approached 1, the pellets were rounder. If the AR reached 1, the pellets were a normal sphere. It can be seen that when the maximum chopper speed was applied, the AR value was lower, these pellets were more spherical (Fig. 6). In our study every sample was spherical or rounder form because the AR values were between 1.18 and 1.37. In the case of high level-high level factor combination (impeller speed: $1500 \mathrm{rpm}$,

Table 5

Coefficients of response surface equations.

\begin{tabular}{|c|c|c|c|c|c|c|c|c|c|}
\hline & $\mathrm{b}_{0}$ & $\mathrm{~b}_{1}(\mathrm{~L})$ & $\mathrm{b}_{1}(\mathrm{Q})$ & $b_{2}(\mathrm{~L})$ & $\mathrm{b}_{2}(\mathrm{Q})$ & $\mathrm{b}_{1}(\mathrm{~L})_{2}(\mathrm{~L})$ & $\mathrm{b}_{1}(\mathrm{~L})_{2}(\mathrm{Q})$ & $\mathrm{b}_{1}(\mathrm{Q})_{2}(\mathrm{~L})$ & $\mathrm{b}_{1}(\mathrm{Q})_{2}(\mathrm{Q})$ \\
\hline $\mathrm{D}_{50}$ & $895.83^{a}$ & $-44.20^{\mathrm{a}}$ & $13.91^{\mathrm{a}}$ & $-1.07^{\mathrm{b}}$ & $48.37^{a}$ & $63.87^{a}$ & $31.09^{a}$ & 12.32 & $45.88^{a}$ \\
\hline A & $55.63^{\mathrm{a}}$ & $-22.22^{\mathrm{a}}$ & $5.21^{\mathrm{a}}$ & $-1.47^{\mathrm{a}}$ & $2.89^{\mathrm{a}}$ & $0.052^{\mathrm{b}}$ & $-2.26^{\mathrm{a}}$ & $8.44^{\mathrm{a}}$ & $-1.81^{\mathrm{a}}$ \\
\hline$\gamma^{\text {tot }}$ & $73.72^{\mathrm{a}}$ & 1.18 & -0.15 & -0.48 & 0.44 & 0.37 & -0.45 & $-0.15^{\mathrm{b}}$ & 0.43 \\
\hline$\gamma^{\mathrm{d}}$ & $40.98^{\mathrm{a}}$ & $1.73^{\mathrm{a}}$ & $-0.51^{\mathrm{a}}$ & $-0.02^{\mathrm{b}}$ & $0.46^{\mathrm{a}}$ & $-1.06^{\mathrm{a}}$ & $0.29^{\mathrm{a}}$ & 0.06 & 0.18 \\
\hline AR & $1.26^{\mathrm{a}}$ & -0.012 & 0.011 & $0.003^{\mathrm{b}}$ & 0.02 & -0.007 & $0.04^{\mathrm{a}}$ & $0.05^{\mathrm{a}}$ & -0.018 \\
\hline $\mathrm{BH}$ & $7.78^{\mathrm{a}}$ & 0.38 & $0.65^{\mathrm{a}}$ & 0.17 & -0.11 & -0.06 & -0.33 & $-0.04^{\mathrm{b}}$ & -0.18 \\
\hline
\end{tabular}

D50: average particle size; A: enzyme activity; $\gamma^{\text {tot. }}$ total surface free energy; $\gamma^{\text {d}}$ : disperse part of surface free energy; AR: aspect ratio; BH: breaking hardness.

a Significant coefficient.

b Ignored coefficient. 
a

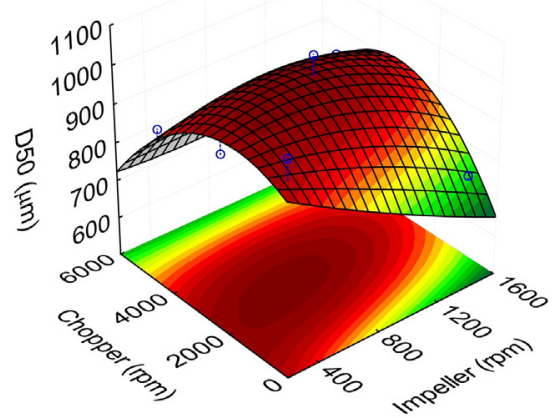

$\mathrm{c}$

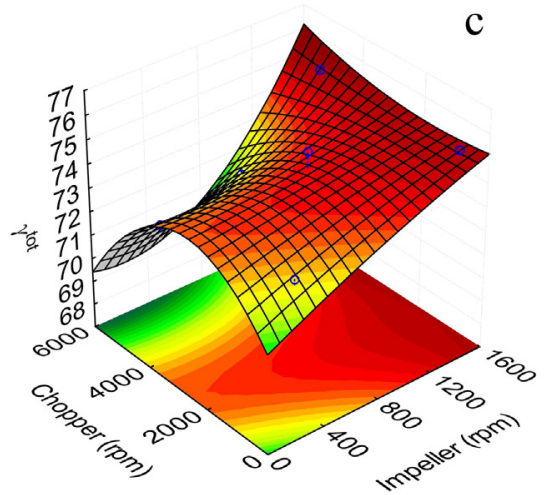

e

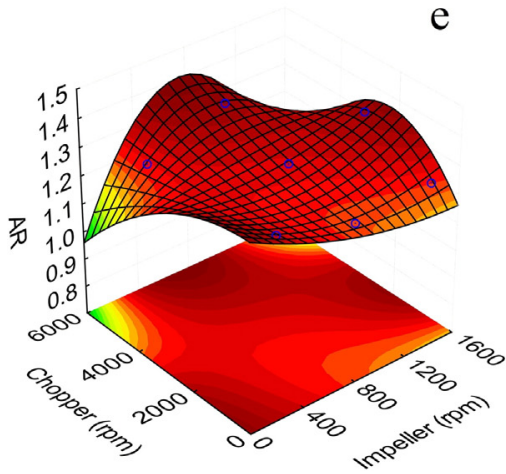

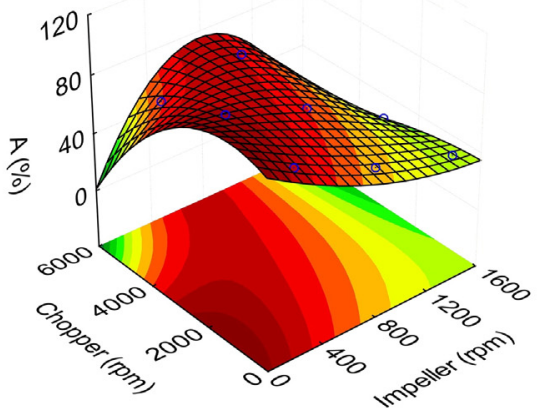

d
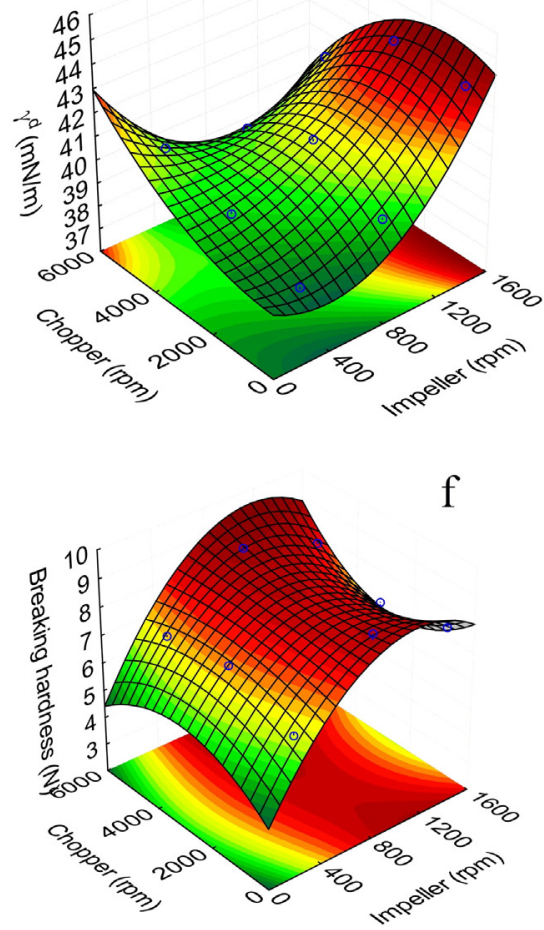

Fig. 6. The response surfaces (a: average particle size; b: enzyme activity; c: surface free energy; d: disperse part of surface free energy; e: aspect ratio; f: breaking hardness).

Table 6

The equation of results of factorial design.

\begin{tabular}{|c|c|}
\hline Parameter & Equations \\
\hline$D_{50}$ & $\begin{array}{l}y=895.83^{\mathrm{a}}-44.2^{\mathrm{a}} x_{1}+13.91^{\mathrm{a}} x_{1}^{2}+48.34^{\mathrm{a}} x_{2}^{2}+63.84^{\mathrm{a}} x_{1} x_{2}+ \\
31.09^{\mathrm{a}} x_{1} x_{2}^{2}+12.32 x_{1}^{2} x_{2}+45.88^{\mathrm{a}} x_{1}^{2} x_{1}^{2}\end{array}$ \\
\hline A & $\begin{array}{l}\mathrm{y}=55.63^{\mathrm{a}}-22.22^{\mathrm{a}} x_{1}+5.21^{\mathrm{a}} x_{1}^{2}-1.47^{\mathrm{a}} x_{2}+2.89^{\mathrm{a}} x_{2}^{2}-2.26^{\mathrm{a}} x_{1} x_{2}^{2}+ \\
8.44^{\mathrm{a}} x_{1}^{2} x_{2}-1.81^{\mathrm{a}} x_{1}^{2} x_{2}^{2}\end{array}$ \\
\hline$\gamma^{\text {tot }}$ & $\begin{array}{l}y=73.72^{\mathrm{a}}+1.18 x_{1}-0.16 x_{1}^{2}-0.48 x_{2}+0.44 x_{2}^{2}+0.37 x_{1} x_{2}-0.45 x_{1} x_{2}^{2}+ \\
0.43 x_{1}^{2} x_{2}^{2}\end{array}$ \\
\hline$\gamma^{\mathrm{d}}$ & $\begin{array}{l}y=40.98^{\mathrm{a}}+1.73^{\mathrm{a}} x_{1}-0.51^{\mathrm{a}} x_{1}^{2}+0.46^{\mathrm{a}} x_{2}-1.06^{\mathrm{a}} x_{1} x_{2}+0.29^{\mathrm{a}} x_{1} x_{2}^{2}+ \\
0.06 x_{1}^{2} x_{2}+0.18 x_{1}^{2} x_{2}^{2}\end{array}$ \\
\hline AR & $\begin{array}{l}\mathrm{y}=1.26^{\mathrm{a}}-0.012 x_{1}+0.011 x_{1}^{2}+0.02 x_{2}^{2}-0.007 x_{1} x_{2}+0.04^{\mathrm{a}} x_{1} x_{2}^{2}+ \\
0.05^{\mathrm{a}} x_{1}^{2} x_{2}-0.018 x_{1}^{2} x_{2}^{2}\end{array}$ \\
\hline $\mathrm{BH}$ & $\begin{array}{l}y=7.78^{\mathrm{a}}+0.38 x_{1}+0.65^{\mathrm{a}} x_{1}^{2}+ \\
0.17 x_{2}-0.11 x_{2}^{2}-0.06 x_{1} x_{2}-0.33 x_{1} x_{2}^{2}-0.18 x_{1}^{2} x_{2}^{2}\end{array}$ \\
\hline
\end{tabular}

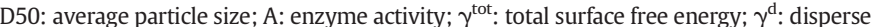
part of surface free energy; AR: aspect ratio; BH: breaking hardness; $x^{1}$ : chopper speed; $x^{2}$ : impeller speed.

a Significant part. chopper speed: $5000 \mathrm{rpm}$ ), the AR was 1.18 and 1.37 in the center point (impeller speed: $900 \mathrm{rpm}$, chopper speed: $2750 \mathrm{rpm}$ ).

The equation of the response surface of AR can be seen in Table 6. In this case only two interaction parts were significant (Table 5). On the response surface it can be seen that only the chopper speed influenced morphology, but in this case the temperature was not increased.

The effect of temperature on the morphology of pellets was not significant (Fig. 7). The impeller speed did not cause a change in the morphology, therefore neither did the temperature. It can be concluded that the elevated temperature during pelletization does not influence the morphology of pellets and a high chopper speed is recommended for spherical form.

\subsection{Mechanical property}

Besides breaking hardness, the deformation process of pellets also gives important information about the mechanical property of pellets. There were three phases. First a short elastic section, a viscoelastic section and a second elastic section up to crunch (first breaking hardness point), a longer elastic section up to the breaking hardness point 

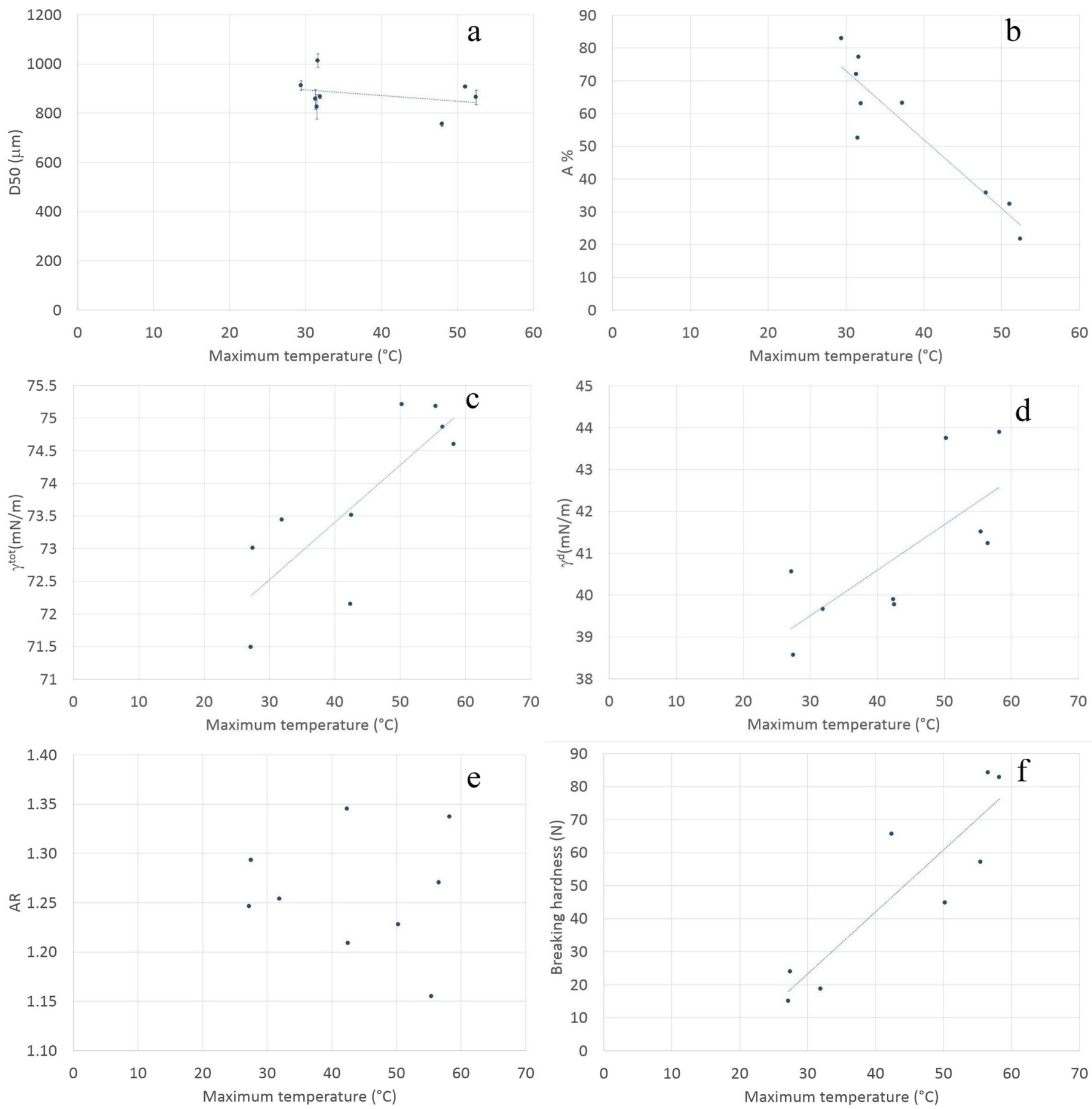

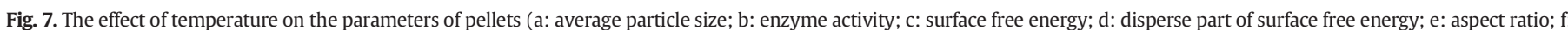
breaking hardness).

(second breaking harness point) (Fig. 9). In our study the deformation processes of all samples were uniform. Only the first and the second breaking hardness points were different.

Table 7

The surface free energy of Vivapur 101 and pepsin.

\begin{tabular}{lll}
\hline Material & $\gamma^{\text {tot }}(\mathrm{mN} / \mathrm{m})$ & $\gamma^{\mathrm{d}}(\mathrm{mN} / \mathrm{m})$ \\
\hline Vivapur 101 & 70.52 & 35.90 \\
Pepsin & 76.11 & 41.28 \\
\hline
\end{tabular}

The breaking hardness can be written with the following equation:

$$
\begin{gathered}
y=7.78^{*}+0.75 x_{1}+1.3^{*} x_{1}{ }^{2}+0.35 x_{2}-0.227 x_{2}{ }^{2} \\
-0.125 x_{1} x_{2}-0.663 x_{1} x_{2}{ }^{2}-0.36 x_{1}{ }^{2} x_{2}
\end{gathered}
$$

In this case only the impeller speed was a significant factor as can be seen in Table 5 and in the equation of the response surface of breaking hardness (Table 6). The chopper speed cannot cause a change in the breaking hardness (Fig. 6). In the literature Mangwandi et al. (2013) found that tensile strength is increasing with increasing impeller 


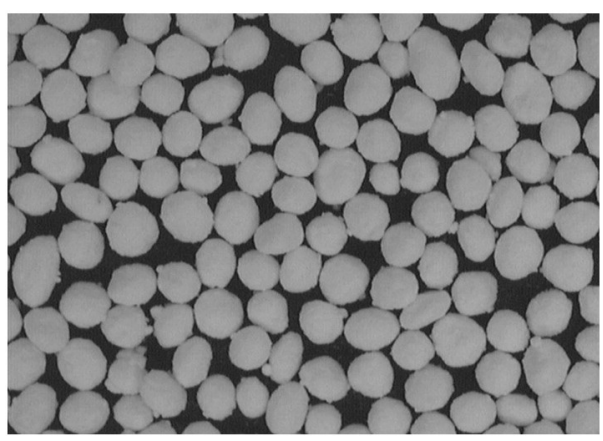

Fig. 8. The morphology of pellets (Zeiss microscope, magnification: $65 \times$ ) (sample prepared at $300 \mathrm{rpm}$ impeller and $5000 \mathrm{rpm}$ chopper speed).

speed. These results correlate with our results. Tensile strength can be calculated from breaking hardness and the pellet size. In our study tensile strength was not calculated because the compound and the size of every sample measured were the same.

Breaking hardness increased with temperature (Fig. 7). If the temperature is elevated during pelletization, the internal energy of this system will also be higher. Therefore the breaking hardness value is also higher. These parameters are very important from the aspect of coating or filling into capsules because the fragmentation of pellets is not allowed.

\section{Conclusion}

The special chamber with PyroButton-TH® and PyroDiff $®$ sensors (Opulus Ltd., Hungary) is appropriate for continuously monitoring temperature and humidity according to PAT. The thermal sensor of the high-shear granulator can measure the temperature only in one point on the surface of the wet masses, therefore it does not provide enough information. The special chamber is appropriate for measuring the temperature inside the chamber in the different regions during granulation. The distribution of temperature was detected inside the chamber during granulation. This information can promote the exact understanding of the granulation process and the optimization steps. The elevated temperature affects the product parameters, enzyme activity, breaking hardness and surface free energy. The relationship between the process parameters and the optimization parameters was determined with factorial design. The high impeller speed causes a high temperature rise,

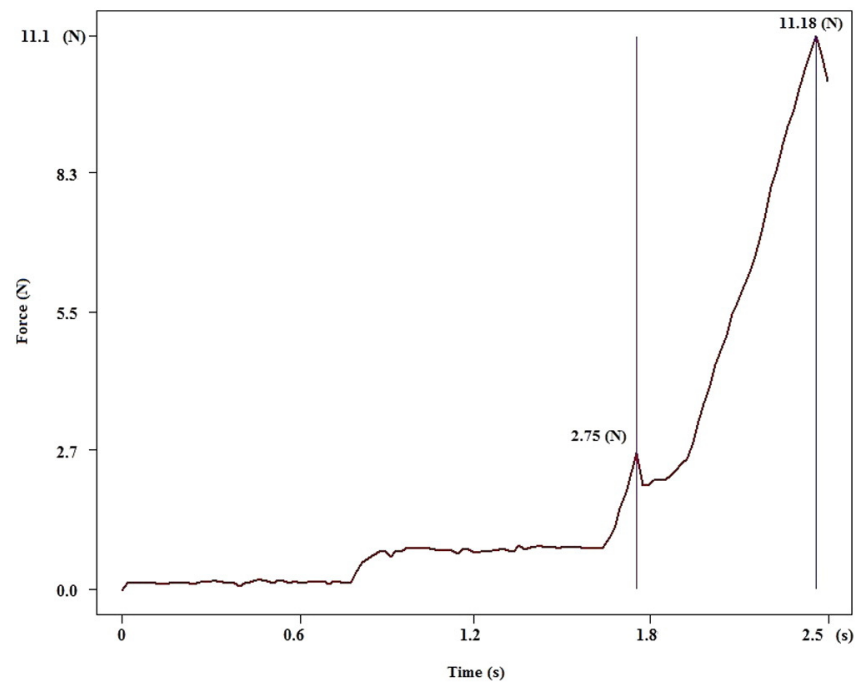

Fig. 9. The deformation process of pellet prepared at 1500 impeller rpm and $500 \mathrm{rpm}$ chopper speed (maximum temperature $50.28^{\circ} \mathrm{C}$ ). which has an effect on the product parameters. During the formulation of granules containing protein, the impeller speed must be taken into consideration because it can decrease the enzyme activity, breaking hardness and the surface free energy. On the basis of our results, the application of high impeller speed is not recommended because the enzyme activity is decreased and the disperse part of surface free energy is increased, which is not favourable from the aspect of coating. The determination of surface free energy can be a useful tool for the prediction of the microstructure of the surface of pellets.

\section{Acknowledgements}

This research was co-financed by the European Union and the State of Hungary (TÁMOP 4.2.4. A/2-11-1-2012-0001 'National Excellence Program').

\section{References}

Bajdik, J., Fehér, M., Pintye-Hódi, K., 2007. Effect of plasticizer on surface of free films prepared from aqueous solutions of salts of cationic polymers with different plasticizers. Appl. Surf. Sci. 253, 7303-7308.

Bakeev, K.A., 2010. Process Analytical Technology: Spectroscopic Tools and Implementation Strategies for the Chemical and Pharmaceutical Industries. 2nd ed. John Wiley \& Sons, Ltd, pp. 1-14.

Benali, M., Gerbaud, V., Hemati, M., 2009. Effect of operating conditions and physicochemical properties on the wet granulation kinetics in high shear mixer. Powder Technol. 190, 160-169.

Bu, D., Wan, B., McGeorge, G., 2013. A discussion on the use of prediction uncertainty estimation of NIR data in partial least squares for quantitative pharmaceutical tablet assay methods. Chemom. Intell. Lab. Syst. 120, 84-91.

Buckton, G., 1997. Characterisation of small changes in the physical properties of powders of significance for dry powder inhaler formulations. Adv. Drug Deliv. Rev. 26, 17-27.

Cárdenas, V., Cordobés, M., Blanco, M., Alcalà, M., 2015. Strategy for design NIR calibration sets based on process spectrum and model space: an innovative approach for process analytical technology. J. Pharm. Biomed. Anal. 114, 28-33.

Chitu, T.M., Oulahna, D., Hemati, M., 2011. Wet granulation in laboratory-scale high shear mixers: effect of chopper presence, design and impeller speed. Powder Technol. 206, 34-43.

Djokića, M., Djuriša, J., Solomuna, L., Kachrimanisb, K., Djurića, Z., Ibrić, S., 2014. The influence of spiral jet-milling on the physicochemical properties of carbamazepine form III crystals: quality by design approach. Chem. Eng. Res. Des. 92, 500-508.

European Pharmacopoeia 8.4, 5.15.

Ferreira, A.P., Tobyn, M., 2015. Multivariate analysis in the pharmaceutical industry: enabling process understanding and improvement in the PAT and QbD era. Pharm. Dev. Technol. 20, 513-527.

Fonteyne, M., Wickström, H., Peeters, E., Vercruysse, J., Ehlers, H., Peters, B.H., Remon, J.P., Vervaet, C., Ketolainen, J., Sandler, N., Rantanen, J., Naelapää, K., De Beer, T., 2014. Influence of raw material properties upon critical quality attributes of continuously produced granules and tablets. Eur. J. Pharm. Biopharm. 87, 252-263.

Guidance for Industry, 2004. PAT - A Framework for Innovative Pharmaceutical Development, Manufacturing, and Quality Assurance.

Hansuld, E.M., Briens, L., 2014. A review of monitoring methods for pharmaceutical wet granulation Int. J. Pharm. 472, 192-201.

Hudovornik, G., Korasa, K., Vrečer, F., 2015. A study on the applicability of in-line measurements in the monitoring of the pellet coating process. Eur. J. Pharm. Sci. 75, $160-168$

ICH Harmonised Tripartited Gudeline, 2009. Pharmaceutical Development Q8 (R2). (http://www.ich.org/fileadmin/Public_Web_Site/ICH_Products/Guidelines/Quality/ Q8_R1/Step4/Q8_R2_Guideline.pdf).

Kaneko, H., Muteki, K., Funatsu, K., 2015. Improvement of iterative optimization technology (for process analytical technology calibration-free/minimum approach) with dimensionality reduction and wavelength selection of spectra. Chemom. Intell. Lab. Syst. 147, 176-184

Kestur, U., Pandey, P., Badawy, S., Lin, J., Desai, D., 2014. Controlling the chemical stability of a moisture-sensitive drug product through monitoring and identification of coating process microenvironment. Int. J. Pharm. 476, 93-98.

Kona, R., Qu, H., Mattes, R., Jancsik, B., Fahmy, R.M., Hoag, S.W., 2013. Application of in-line near infrared spectroscopy and multivariate batch modeling for process monitoring in fluid bed granulation. Int. J. Pharm. 452, 63-72.

Kristó, K., Pintye-Hódi, K., 2013. Effects of pharmaceutical processing on pepsin activity during the formulation of solid dosage forms. Pharm. Dev. Technol. 18, 17-21.

Lopesa, J.A., Costa, P.F., Alves, T.P., Menezes, J.C., 2004. Chemometrics in bioprocess engineering: process analytical technology (PAT) applications. Chemom. Intell. Lab. Syst. 74, 269-275

Luukkonen, P., Fransson, M., Niklasson, I.B., Hautala, J., Lagerholm, B., Folestad, S., 2008. Real-time assessment of granule and tablet properties using in-line data from a high-shear granulation process. J. Pharm. Sci. 99, 950-959.

Macchi, E., Zema, L., Pandey, P., Gazzaniga, A., Felton, L.A., 2016. Influence of temperature and relative humidity conditions on the pan coating of hydroxypropyl cellulose molded capsules. Eur. J. Pharm. Biopharm. 100, 47-57. 
Mangwandi, C., Adams, M.J., Hounslow, M.J., Salman, A.D., 2010. Effect of impeller speed on mechanical and dissolution properties of high-shear granules. Chem. Eng. J. 164, 305-315.

Mangwandi, C., Tao, L.J., Albadarin, A.B., Allen, S.J., Walker, G.M., 2013. Alternative method for producing organic fertiliser from anaerobic digestion liquor and limestone powder: high shear wet granulation. Powder Technol. 233, 245-254.

Pandey, P., Bindra, D.S., Gour, S., Trinh, J., Buckley, D., Badawy, S., 2014a. Excipient-process interactions and their impact on tablet compaction and film coating. J. Pharm. Sci. 103, 3666-3674.

Pandey, P., Bindra, D.S., Felton, L.A., 2014b. Influence of process parameters on tablet bed microenvironmental factors during pan coating. AAPS PharmSciTech 15, 296-305.

Rahmanian, N., Naji, A., Ghadiri, M., 2011. Effects of process parameters on granules properties produced in high shear granulator. Chem. Eng. Res. Des. 89, 512-518.

Rantanen, J., Knskoski, M., Suhonen, J., Tenhunen, J., Lehtonen, S., Rajalahti, T., Mannermaa, J.P., Yliruusi, J., 2000. Next generation fluidized bed granulator automation. AAPS PharmSciTech 1, 26-36.

Rantanen, J., Jørgensen, A., Räsänen, E., Luukkonen, P., Airaksinen, S., Raiman, J., Hänninen, K., Antikainen, O., Yliruusi, J., 2001. Process analysis of fluidized bed granulation. AAPS PharmSciTech 2, 13-20.
Reid, G.L., Ward, H.W., Palm., A.S., Muteki, K., 2012. Process analytical technology (PAT) in pharmaceutical development. Am. Pharm. Rev. 15, 49-55.

Roggo, Y., Chalus, P., Maurer, L., Martinez, C.L., Edmond, A., Jent, N., 2007. A review of near infrared spectroscopy and chemometrics in pharmaceutical technologies. J. Pharm. Biomed. 44, 683-700.

Rowe, R.C., 1989. Binder-substrate interactions in granulation: a theoretical approach based on surface free energy and polarity. Int. J. Pharm. 52, 149-154.

Shah, B., Khunt, D., Bhatt, H. Misra, M., Padh, H., 2015. Application of quality by design approach for intranasal delivery of rivastigmine loaded solid lipid nanoparticles: effect on formulation and characterization parameters. Eur. J. Pharm. Sci. 78, 54-66.

Simon, L.M., Kotormán, M., Szabó, A., Nemcsók, J., Laczkó, I., 2007. The effects of organic solvent/water mixtures on the structure and catalytic activity of porcine pepsin. Process Biochem. 42, 909-912.

Wu, S., 1971. Calculation of interfacial tension in polymer systems. J. Polym. Sci. 34, 19-30

Zeng, H., You, J., Liang, H., Qi, T., Yang, R., Qu, L., 2014. Investigation on the binding interaction between silybin and pepsin by spectral and molecular docking. Int. J. Biol. Macromol. 67, 105-111.

Žižek, K., Hraste, M., Gomzi, Z., 2013. High shear granulation of dolomite - I: effect of shear regime on process kinetics. Chem. Eng. Res. Des. 91, 70-86. 